\title{
THE EQUIVALENCE OF THE ANNULUS CONJECTURE AND THE SLAB CONJECTURE ${ }^{1}$
}

\author{
BY CHARLES GREATHOUSE
}

Communicated by M. L. Curtis, May 29, 1964

In [1], the author showed that the Slab Conjecture implies the Annulus Conjecture.

The purpose of this paper is to show that the Annulus Conjecture implies the Slab Conjecture for $n>3$ and hence the two conjectures are equivalent for $n>3$.

$R^{n}, S^{n}$ will denote $n$-space and the $n$-sphere, respectively. A $k$ manifold $N$ is embedded in a locally flat manner in an $n$-manifold $M$ provided each point of $N$ has a neighborhood $U$ in $M$ such that $(U, U \cap N) \approx\left(R^{n}, R^{k}\right)$.

The Annulus Conjecture. Let $S_{1}^{n-1}, S_{2}^{n-1}$ be disjoint locally flat $(n-1)$-spheres embedded in $S^{n}$ and let $M$ be the submanifold of $S^{n}$ bounded by $S_{1}^{n-1} \cup S_{2}^{n-1}$. Then $M \approx S^{n-1} \times[0,1]$.

The Slab Conjecture. Let $R_{1}^{n-1}, R_{2}^{n-1}$ be disjoint locally flat $n-1$ spaces embedded as closed subsets of $R^{n}$ and let $M$ be the submanifold of $R^{n}$ bounded by $R_{1}^{n-1} \cup R_{2}^{n-1}$. Then $M \approx R^{n-1} \times[0,1]$.

Theorem. The Annulus Conjecture implies the Slab Conjecture for $n>3$.

PRoof. Let $R_{1}^{n-1}, R_{2}^{n-1}$ be disjoint locally flat $n-1$ spaces embedded as closed subsets of $R^{n}, n>3$, and let $M$ be the submanifold of $R^{n}$ bounded by $R_{1}^{n-1} \cup R_{2}^{n-1}$. Let $S^{n}=R^{n} \cup\{p\}$ be the one-point compactification of $R^{n}$ and $S_{i}^{n-1}=R_{i}^{n-1} \cup\{p\}$ for $i=1,2$. By the corollary to Theorem 2 of [2], $S_{i}^{n-1}$ is flat for $i=1,2$. Hence, we may assume that $S_{1}^{n-1}=S^{n-1}$, that $S_{2}^{n-1}$ lies in the northern hemisphere of $S^{n}=$ the suspension of $S^{n-1}$, and that $S_{1}^{n-1} \cap S_{2}^{n-1}=\{p\}$.

Let $B^{n-1}$ be the unit ball in $S_{1}^{n-1}=S^{n-1}$ with center $p, r=$ the south pole of $S^{n}, q=$ the midpoint of the line segment joining $p$ to $r$ in $S^{n}$, $L=$ the line segment joining $p$ to $q$ in $S^{n}$, and $B_{r}^{n}, B_{q}^{n}=$ the cones ( $n$-balls) in $S^{n}$ with bases $B^{n-1}$ and cone points $r, q$ respectively. (See Figure 1.) Now, let $S_{3}^{n-1}=\left[S_{1}^{n-1} \cup \dot{B}_{q}^{n}\right]-\operatorname{Int}\left(B^{n-1}\right)$. Then $S_{3}^{n-1}$ is a flat $n-1$ sphere in $S^{n}$ and $S_{3}^{n-1} \cap S_{2}^{n-1}=\varnothing$. By the Annulus Conjecture, $M \cup B_{q}^{n}=A^{n}$ is an $n$-annulus. We will complete the proof by showing that $M \cup\{p\}$ is homeomorphic to the decomposition space $A^{n} / L$ and applying Lemma 3 of [3].

1 This work supported in part by NSF GP-211. 


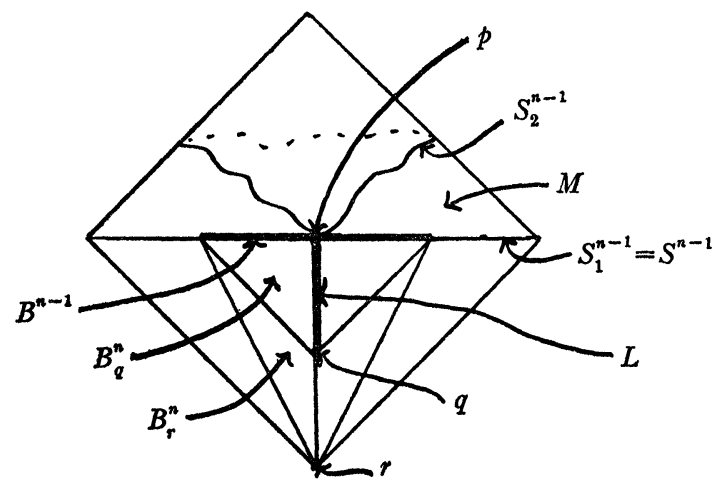

Figure 1

By Theorem II.3 of [1], $M_{2}=M-R_{2}^{n-1} \approx R^{n-1} \times[0,1)$ under some homeomorphism $h$. Take $T=h^{-1}\left[h\left(B^{n-1}-p\right) \times\left[0, \frac{1}{2}\right]\right], T_{r}=T \cup B_{r}^{n}$, and $T_{q}=T \cup B_{q}^{n}$. Then $T_{r}, T_{q}$ are $n$-balls with $T_{q} \subset T_{r}$.

There is a natural map $f$ of $T_{r}$ onto itself such that the following hold:

(1) $f \mid \dot{T}_{r}=1$,

(2) $f \mid T_{r}-L$ is a homeomorphism,

(3) $f(L)=p$,

(4) $f\left[\mathrm{CL}\left(B_{r}^{n}-B_{q}^{n}\right)\right]=B_{r}^{n}$.

$f$ is obtained by pushing $B_{q}^{n}$ up into $T \cup\{p\}$ making use of the parameterization induced on $T$ by $h^{-1} \cdot f$ extends to a map of $S^{n}$ onto itself by $f \mid S^{n}-T_{r}=1$.

Since $f\left(A^{n}\right)=M \cup\{p\}, f \mid A^{n}-L$ is a homeomorphism and $f(L)=p$, it follows that $M \cup\{p\} \approx A^{n} / L$. By Lemma 3 of [3], since $L$ is a flat arc in $A^{n}$ with endpoints $p \in S_{2}^{n-1}, q \in S_{3}^{n-1}$ and $L-(p \cup q) \subset \operatorname{Int} A^{n}$, $A^{n} / L$ is a pinched annulus, that is, $A^{n} / L$ is homeomorphic to the onepoint compactification of $R^{n-1} \times[0,1]$. Thus $M \approx R^{n-1} \times[0,1]$ and the theorem is proved.

Corollary. The Annulus Conjecture is equivalent to the Slab Conjecture for $n>3$.

\section{REFERENCES}

1. Charles Greathouse, Locally fat strings, Bull. Amer. Math. Soc. 70 (1964), 415-418.

2. J. C. Cantrell, Almost locally flat embeddings of $S^{n-1}$ in $S^{n}$, Bull. Amer. Math. Soc. 69 (1963), 716-718.

3. - Some relations between the Annulus Conjecture and union of fat cells theorems (to appear).

UnIVERSiTy OF TENNESSEE 